\title{
RELATÓRIO DA GESTÃO 2009
}

\author{
Silvana Calvo Tuleski \\ Marta Eliane Echeverria Borges ${ }^{\#}$
}

O presente relatório objetiva descrever a gestão editorial da revista Psicologia em Estudo do ano de 2009. Atualmente a revista tem periodicidade trimestral e conta com catorze volumes publicados, contabilizados a partir de sua origem em 1996, quando veio a público o Vol. 1, n. 1, sob editoração geral da professora doutora Maria Lúcia Boarini, do Departamento de Psicologia da Universidade Estadual de Maringá.

Desde o período de sua fundação a revista vem procurando aperfeiçoar e melhorar seu processo de editoração e a qualidade das publicações. Para retratar brevemente o seu crescimento, destaca-se o fato de que em seus primeiros cinco anos de existência a revista publicava, em média, dez artigos por número e sua periodicidade era quadrimestral. A partir de 2001 este quadro começou a se alterar com o crescimento do número de artigos enviados para o periódico, que foi alcançando cada vez maior visibilidade em nível nacional e internacional, ainda sob a editoração de sua fundadora.

No último triênio que antecede este relatório, a revista Psicologia em Estudo passou a ter como editora geral a professora doutora Rozilda das Neves Alves, que deu continuidade a um trabalho árduo de dez anos da editora fundadora.

Desde o seu início, o apoio e parceria em infraestrutura para parte do processo editorial, preparação, distribuição e indexação dos volumes adveio da Universidade Estadual de Maringá, do Departamento de Psicologia e, mais recentemente, em 2008, do Programa de Pós-Graduação em Psicologia da mesma instituição.

Entre os anos de 2008 e 2009 houve novas alterações na equipe da Revista, decorrentes de inúmeros fatores, entre eles a perspectiva de aposentadoria de alguns professores; porém essa transição vem sendo realizada sem perder de vista os cuidados arrolados com a editoração da Psicologia em Estudo. Dos membros que compunham a equipe em 2008, alguns permaneceram e permanecerão como integrantes em 2010, contribuindo para que se mantenha a qualidade até então garantida. Os novos membros são professores da graduação e pós-graduação do Departamento de Psicologia da Universidade Estadual de Maringá, tendo já participado de elaborações anteriores, como consultores ad hoc ou mesmo como assistentes de editoração.

Vale ressaltar que em 2009 a revista obteve recursos do Programa de Apoio a Publicações Científicas do Conselho Nacional de Desenvolvimento Cientifico e Tecnológico (CNPq) e da Fundação Araucária de Apoio ao Desenvolvimento Científico e Tecnológico do Paraná, o que muito auxiliou na manutenção da qualidade dos procedimentos editoriais.

Desde o ano de 2001, a partir do volume 6, número 2, a Revista passou a disponibilizar seus artigos na íntegra pelo Sistema Scielo de publicação (Scientific Electronic Library Online) e na base de dados Scopus Elsevier. Também está indexada em bases de dados referenciais que contêm os dados essenciais dos artigos, como: LILACS (Literatura Latino-Americana e do Caribe em Ciências da Saúde), do Centro Latino-Americano e do Caribe de Informações em Ciências da Saúde; Index Psi Periódicos, resultante de parceria entre o Conselho Federal de Psicologia e a Pontifícia Universidade Católica de Campinas; CLASE (Citas Latinoamericanas En Ciencias Sociales y Humanidades), gerida pela UNAM

Doutora em Educação Escolar, Docente do Departamento de Psicologia da Universidade Estadual de Maringá, Editora da revista Psicologia em Estudo.

\# Doutoranda em Desenvolvimento de Processos no programa de pós-graduação de Engenharia Química da UEM, membro da comissão executiva da revista Psicologia em Estudo. 
(Universidad Nacional Autónoma de México); Sociological Abstracts (Cambridge Scientific Abstracts); Social Services Abstracts, e; Linguistics \& Language Behavior Abstracts.

No que tange à avaliação dos periódicos científicos da área, a revista Psicologia em Estudo tem sido analisada pelos critérios instituídos pelo Qualis de Periódicos, na área de Psicologia, da comissão conjunta Capes-Anpepp (Coordenação de Aperfeiçoamento de Pessoal de Nível Superior/Associação Nacional de Pesquisa e PósGraduação em Psicologia), desde 2005, ano em foi classificada como Nacional A. Em 2007, a partir da análise dos números editados em 2005 e 2006, foi classificada como Internacional A. Em 2008 os critérios elaborados por essa comissão foram revistos, passando-se a dar grande relevância à indexação dos periódicos em bases de dados reconhecidas internacionalmente, sendo que as publicações foram hierarquizadas em estratos (A1, A2; B1, B2, B3, B4, B5) e a revista Psicologia em Estudo foi classificada no estrato A2.

$\mathrm{O}$ processo de arbitragem dos manuscritos submetidos à Psicologia em Estudo pauta-se no critério de revisão cega (Double blind review) por pares da comunidade científica, ou seja, tanto as identidades de autores como as de assessores ad hoc mantêm-se incógnitas. A tramitação editorial desta revista é resumida na figura 1.

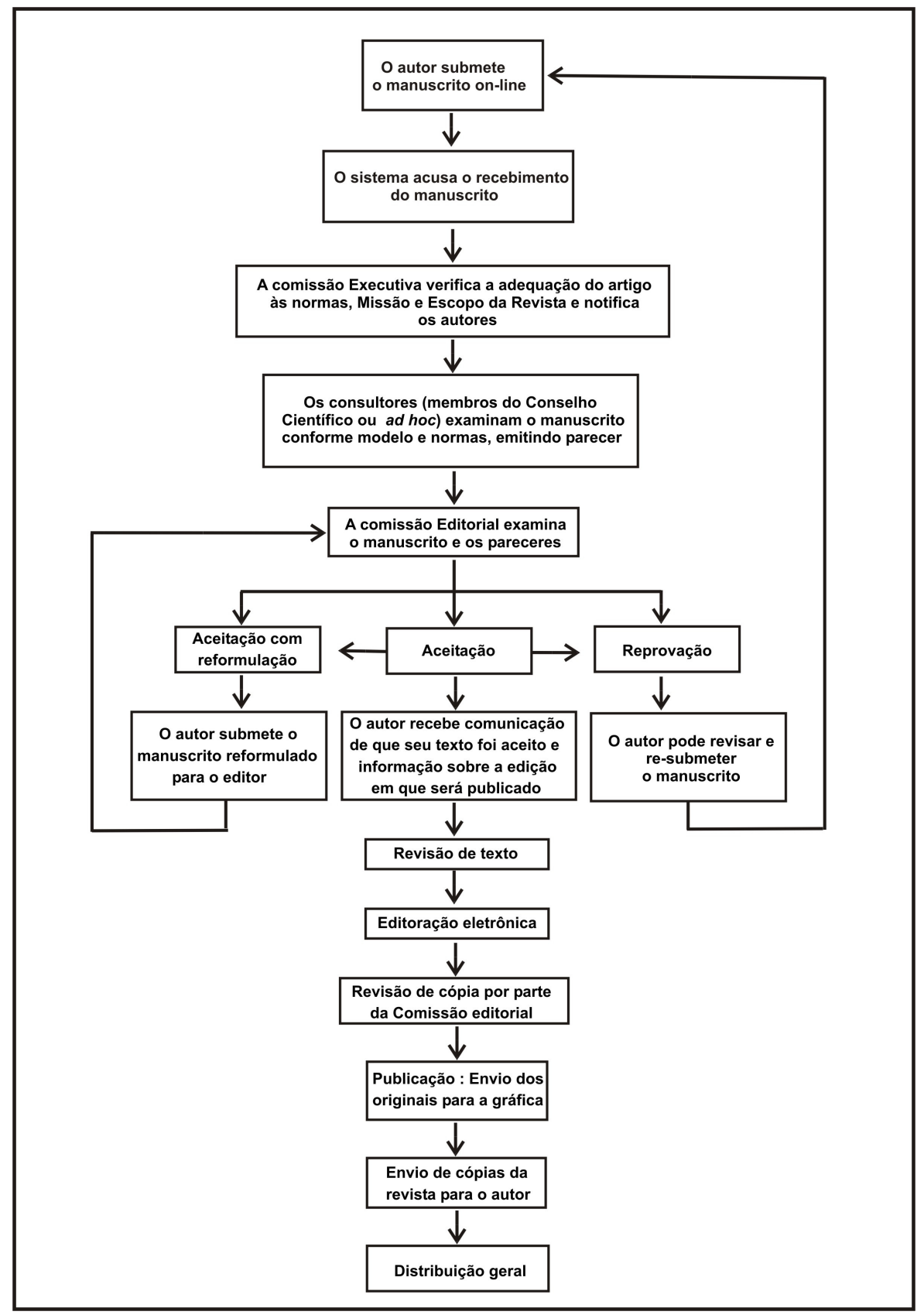

Figura 1. Fluxograma de Tramitação de Artigos 
Destaca-se que este periódico contempla em suas edições textos de autores de diferentes universidades nacionais e internacionais, além de profissionais de outras instituições, e tem como objetivo principal incentivar a produção e a divulgação de conhecimentos referentes à Psicologia e áreas afins, promovendo o intercâmbio pedagógico e científico entre professores, acadêmicos e profissionais.

A análise dos dados referentes ao trabalho editorial de 2009, efetuada no primeiro bimestre de 2010, revelou a tramitação do total de trezentos e quarenta e quatro artigos (em papel e on-line), somados os pendentes de 2006 a 2008. Dos artigos antigos, cujo controle ainda era realizado de modo impresso, tivemos dois artigos de 2006 (um publicado em 2009 e um a ser publicado em 2010), cinquenta e oito artigos de 2007 (oito arquivados por não retorno dos autores, quarenta e nove publicados em 2009 e um a ser publicado em 2010), quinze artigos de 2008 (dois arquivados por não retorno dos autores e treze publicados em 2009). Dos artigos que tramitaram pelo sistema on-line em 2009, oitenta e um foram submetidos em 2008 (destes, quarenta e dois foram arquivados, vinte e seis foram aprovados para publicação e treze ainda estão em avaliação por consultores). Em 2009, dos cento e oitenta e oito artigos submetidos no sistema on-line, cento e dois foram arquivados, sete estão aprovados para publicação até o momento e setenta e nove encontram-se em avaliação por consultores.

Dos artigos que tramitaram no sistema on-line em 2009, considerando os submetidos no segundo semestre de 2008 e os de todo o ano de $2009^{1}$, encontramos a classificação segundo a modalidade de submissão, apresentada na figura 2 , a seguir.

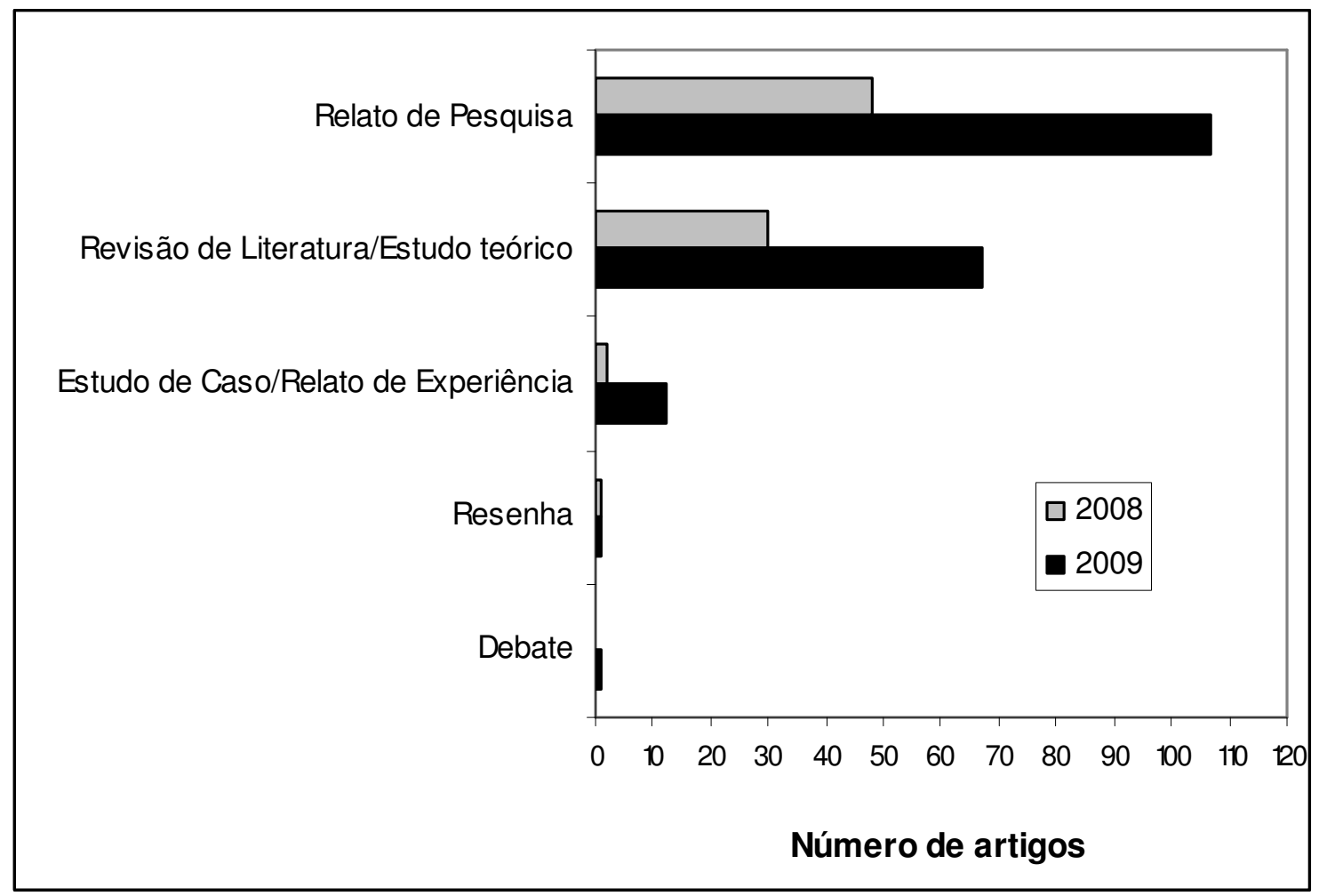

Figura 2. Modalidades dos Artigos que Tramitaram no Sistema On-line em 2009

\footnotetext{
1 Destaca-se que no segundo semestre de 2008 iniciou-se o processo de submissão dos artigos pelo sistema on-line, mas estas submissões eram realizadas, em sua maioria, pela Equipe Executiva da revista, pois ainda havia um grande número de artigos impressos recebidos pelo correio. Em janeiro de 2009 as submissões passaram a ser realizadas integralmente pelos autores, a partir do sistema on-line.
} 
Assim, em 2009 foram publicados, ao todo, oitenta e quatro artigos, dos quais oitenta são relatos de pesquisa/estudos teóricos, três são relatos de experiência profissional e um é resenha - além dos editoriais, que não entram no cômputo geral, por sua natureza diversa. Verifica-se que há quantidade significativa de submissões de relatos

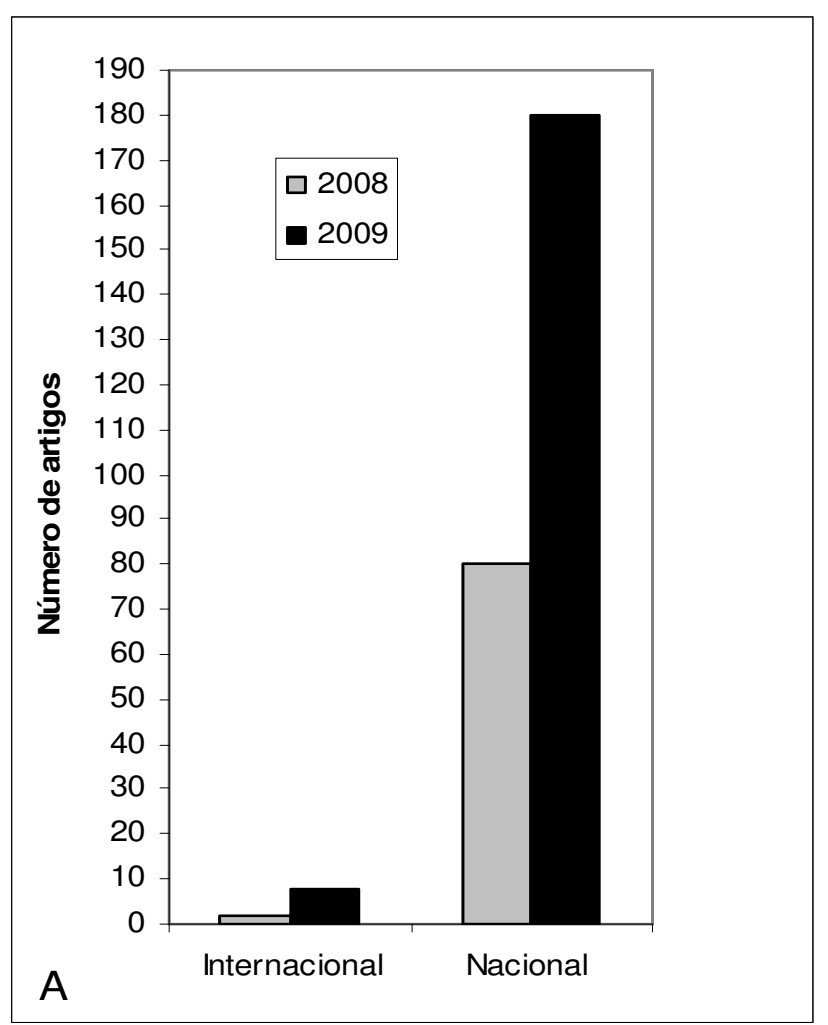

de pesquisa e revisão de literatura/estudo teórico, em comparação com resenhas, estudos de caso e debates.

A distribuição dos artigos que tramitaram no sistema on-line de acordo com a região (internacional/nacional/estados) pode ser verificada na figura 3.

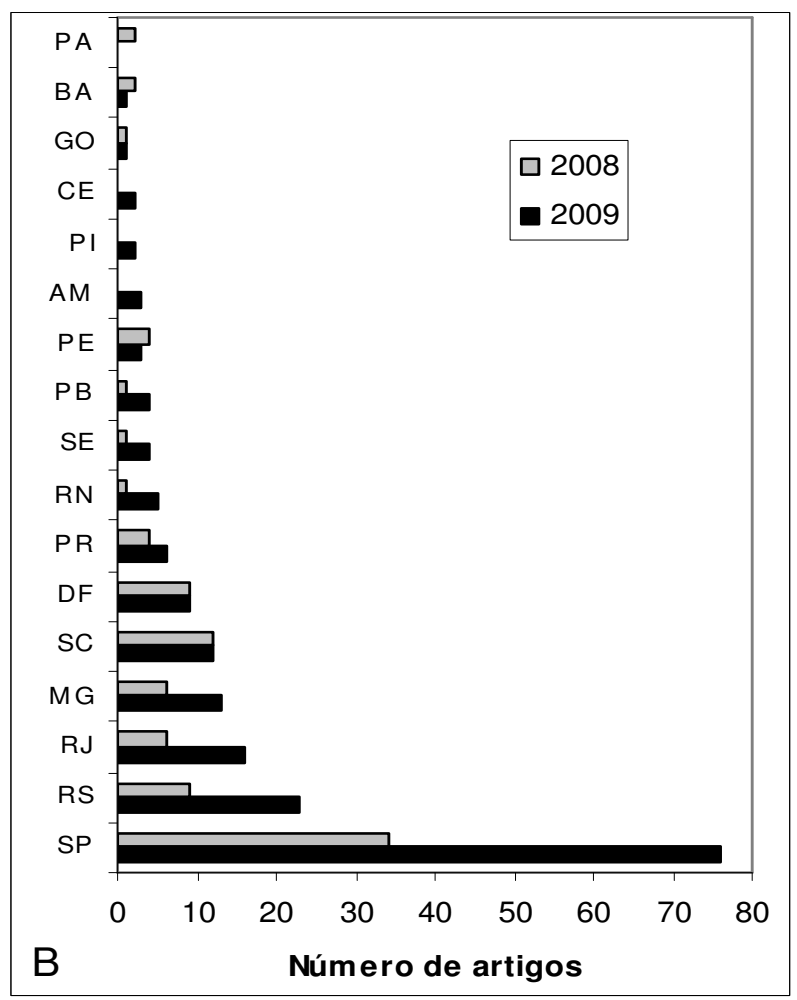

Figura 3. Número de Artigos que Tramitaram no Sistema On-line em 2009 Segundo os Âmbitos Nacional e Internacional (A) e os estados Brasileiros (B).

Quanto aos artigos em avaliação que tramitaram on-line em 2009 (excluídos os arquivados), de acordo com a instituição de filiação dos autores nacionais, pode-se constatar que as instituições que mais contribuíram com artigos são das regiões Sudeste e Sul, bem como houve a predominância da submissão de artigos de universidades públicas em comparação com as privadas (figuras 4 e 5), o que também se observa em outros periódicos.
Em relação ao número de artigos submetidos desde 2007, pode-se constatar que em 2009 houve o maior número de artigos submetidos e o maior número de artigos pendentes, quando somados os artigos submetidos em papel e aqueles on-line (figura 6). 


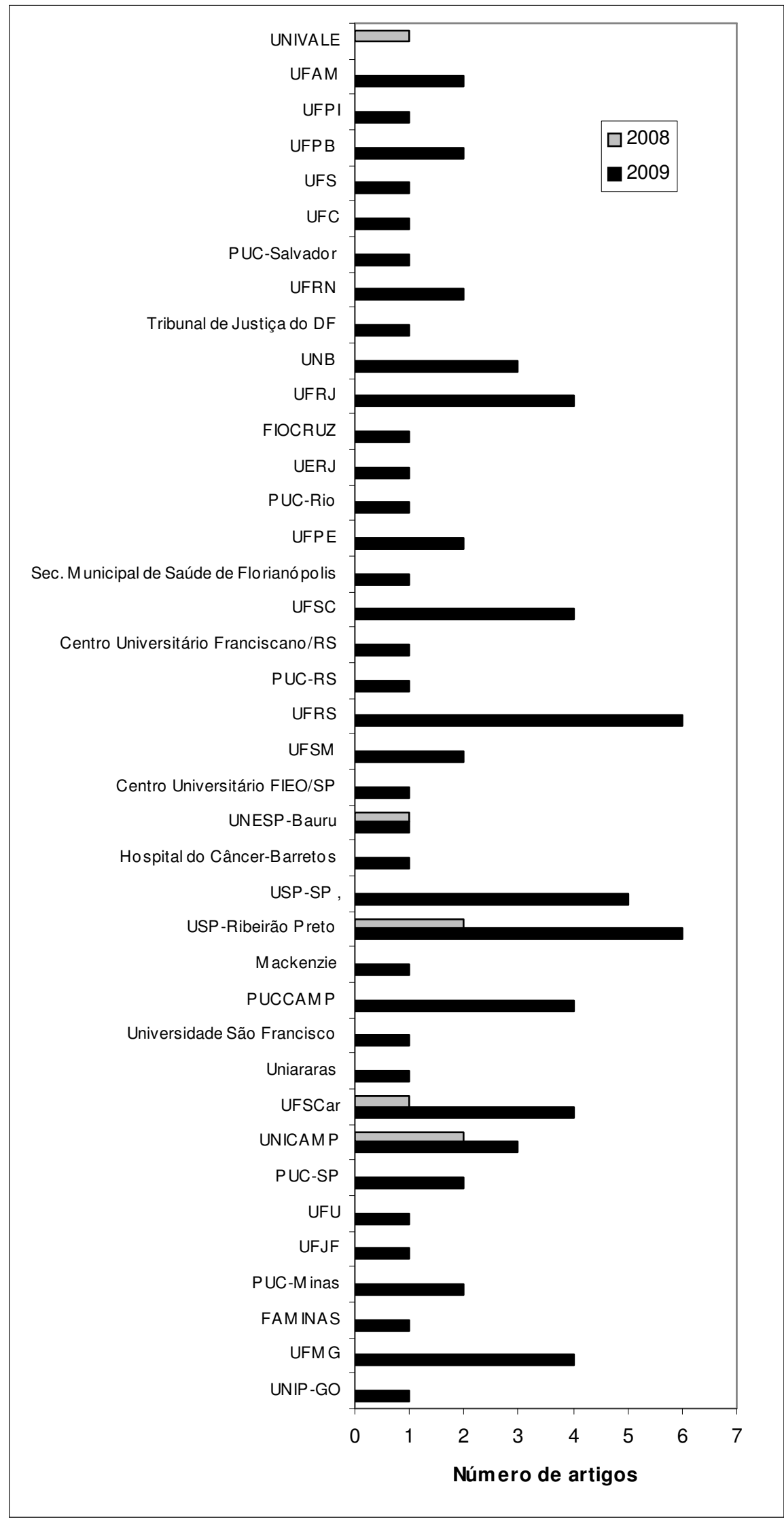

Figura 4. Número de Artigos em Avaliação que Tramitaram On-line em 2009, Referente a Instituições Nacionais 


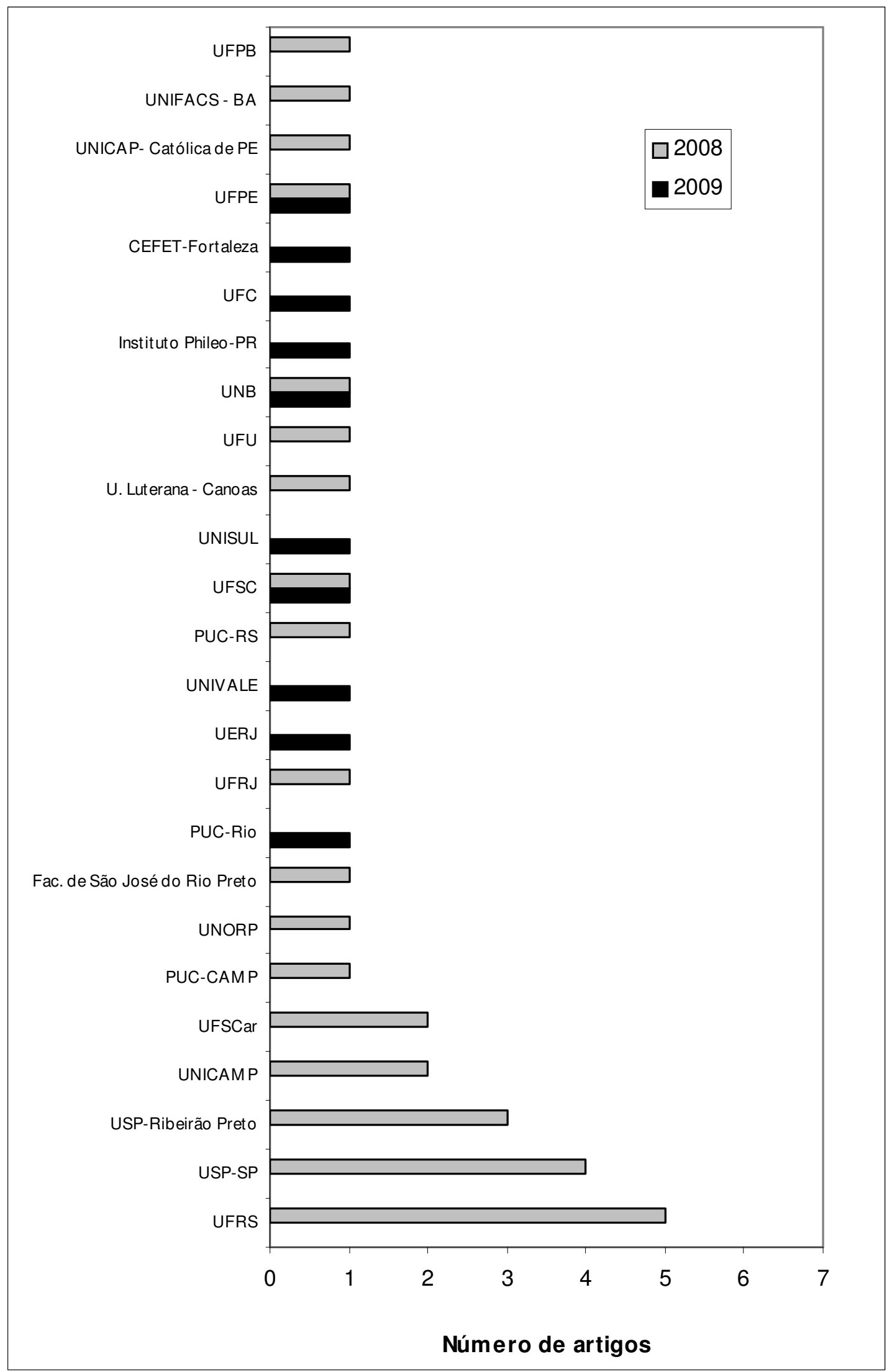

Figura 5. Número de Artigos em Edição que Tramitaram on-line em 2009, Referente a Instituições Nacionais 

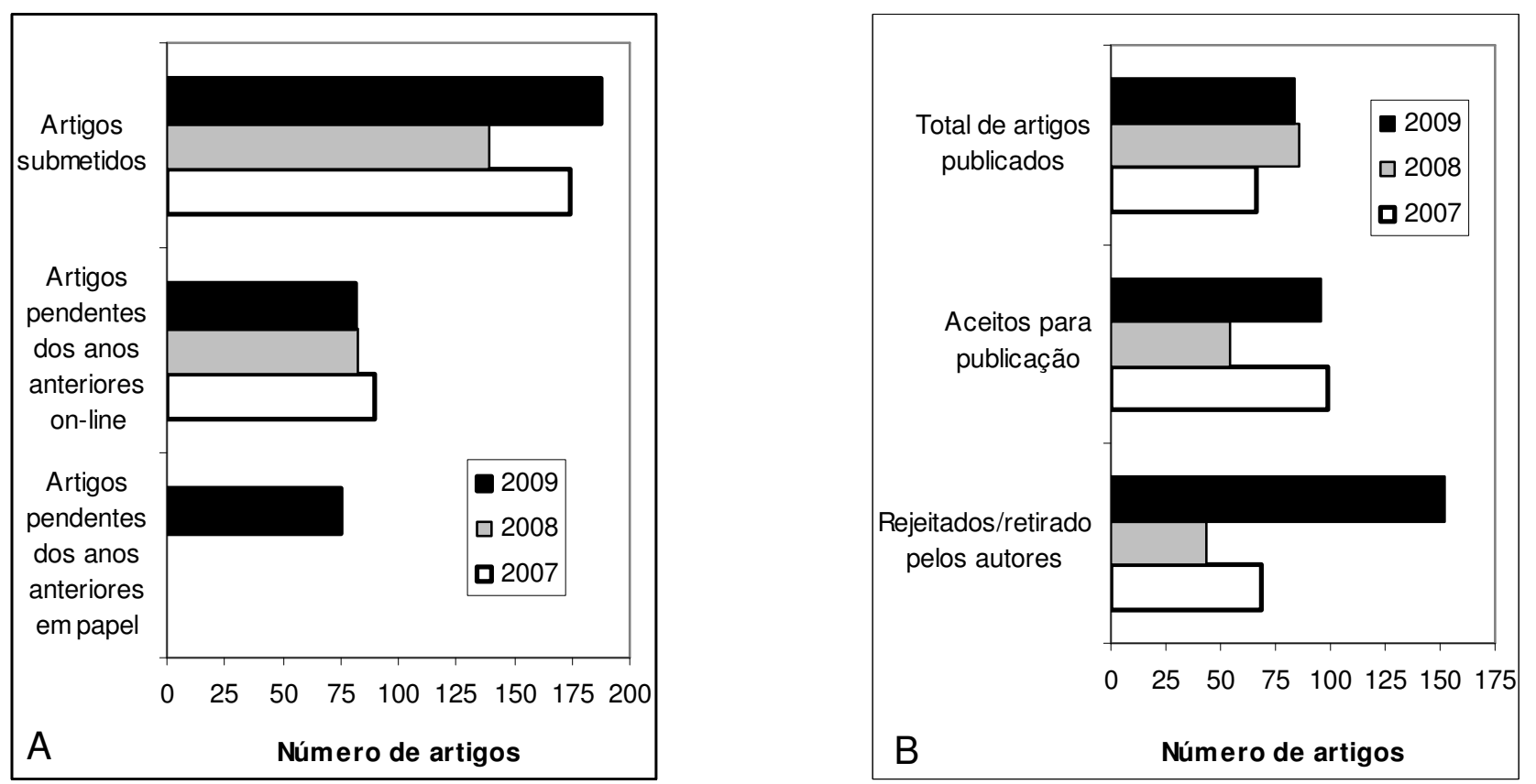

Figura 6. Número de Artigos Submetidos e Pendentes nos Últimos Três Anos (A) e Número de Artigos trabalhados (B).

O número de artigos pendentes tem dificultado a rapidez na publicação. Isto pode ser observado nas figuras 6 e 7, as quais mostram que o ano de 2008 teve o menor número de artigos submetidos e o menor período para a aprovação do artigo; porém nesse ano registrou-se o maior tempo de espera para publicação, pelo fato de o ano de 2007, embora tenha tido o maior número de artigos aceitos, foi o ano em que se publicaram menos artigos correspondentes àquele ano. Assim, o número de artigos pendentes correspondentes ao período de 2006 a 2008 que foram publicados em 2009 dificultou grande parte da publicação dos artigos submetidos neste último ano, constatando-se que o período entre submissão e publicação aumentou para 20 meses.

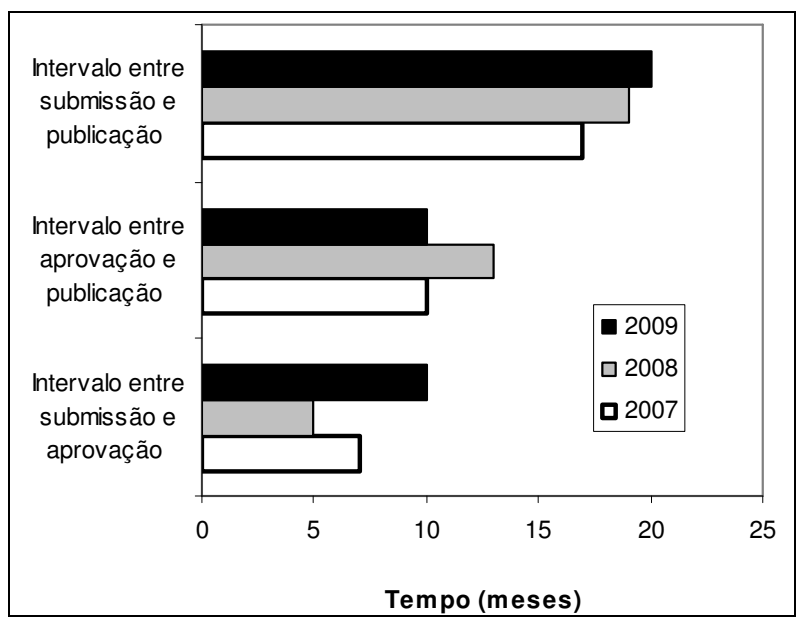

Figura 7. Período de Tramitação dos Artigos
Por uma tendência do meio editorial científico, ante as demandas já citadas que se apresentaram e as condições criadas, a partir de 2004 a periodicidade da revista Psicologia em Estudo tornou-se quadrimestral e em 2008 passou a ser trimestral. É importante destacar que parte desta alteração decorreu do fluxo de artigos que são submetidos e tramitam no periódico, que a cada ano tem aumentado e, no ano de 2009, devido à implementação das submissões somente online, teve um salto significativo em relação a 2008: aproximadamente $35 \%$.

Com a trimestralidade da Revista a partir de 2008 - que aumentou o custo anual do periódico - obteve-se a publicação de uma média de 21 artigos por número, o que ainda não foi suficiente para reduzir o acúmulo gradativo, principalmente pela demanda aumentada com as submissões on-line. Entende-se que o aumento das submissões de artigos à revista Psicologia em Estudo é fruto de sua visibilidade nacional e internacional e da excelente avaliação que o periódico obteve junto à Capes/Anpepp; porém, contraditoriamente, o aumento da demanda ocasiona dificuldades crescentes à vazão dos artigos aceitos para publicação, que exige da Equipe Executiva a criação de mecanismos para a seleção dos artigos que tramitarão no periódico.

Em decorrência do esforço de sua equipe em atender às exigências de qualidade e respeitabilidade científicas, regulamentadas como exposto, a revista Psicologia em Estudo, no ano de 2009, enfrentou diversos desafios, quando as submissões passaram a ser efetuadas somente via on-line, o que exigiu 
readequação das normas objetivando maior clareza aos leitores, autores e avaliadores ad hoc e ajustamento de funções na equipe executiva para que a operacionalização contasse com maior objetividade e agilidade dos processos editoriais. Ademais, foi necessário gerar procedimentos para a publicação dos artigos aprovados e pendentes de anos anteriores, para diminuir o tempo entre submissão e publicação.

Um dos procedimentos adotados para que não se repita o acúmulo de artigos de anos anteriores acúmulo que estende o prazo entre submissão e publicação - foi estabelecer suspensões temporárias para novas submissões no sistema on-line. Outra ação adotada pela Equipe Executiva foi uma maior exigência em relação aos artigos submetidos no que tange à sua adequação às normas editoriais e à missão e escopo da revista, bem como em relação à qualidade e originalidade desses artigos.

É importante destacar que tais exigências levaram ao aumento do número de artigos arquivados em 2009, por não se adequarem às normas editoriais, como pode ser constatado na figura 8.

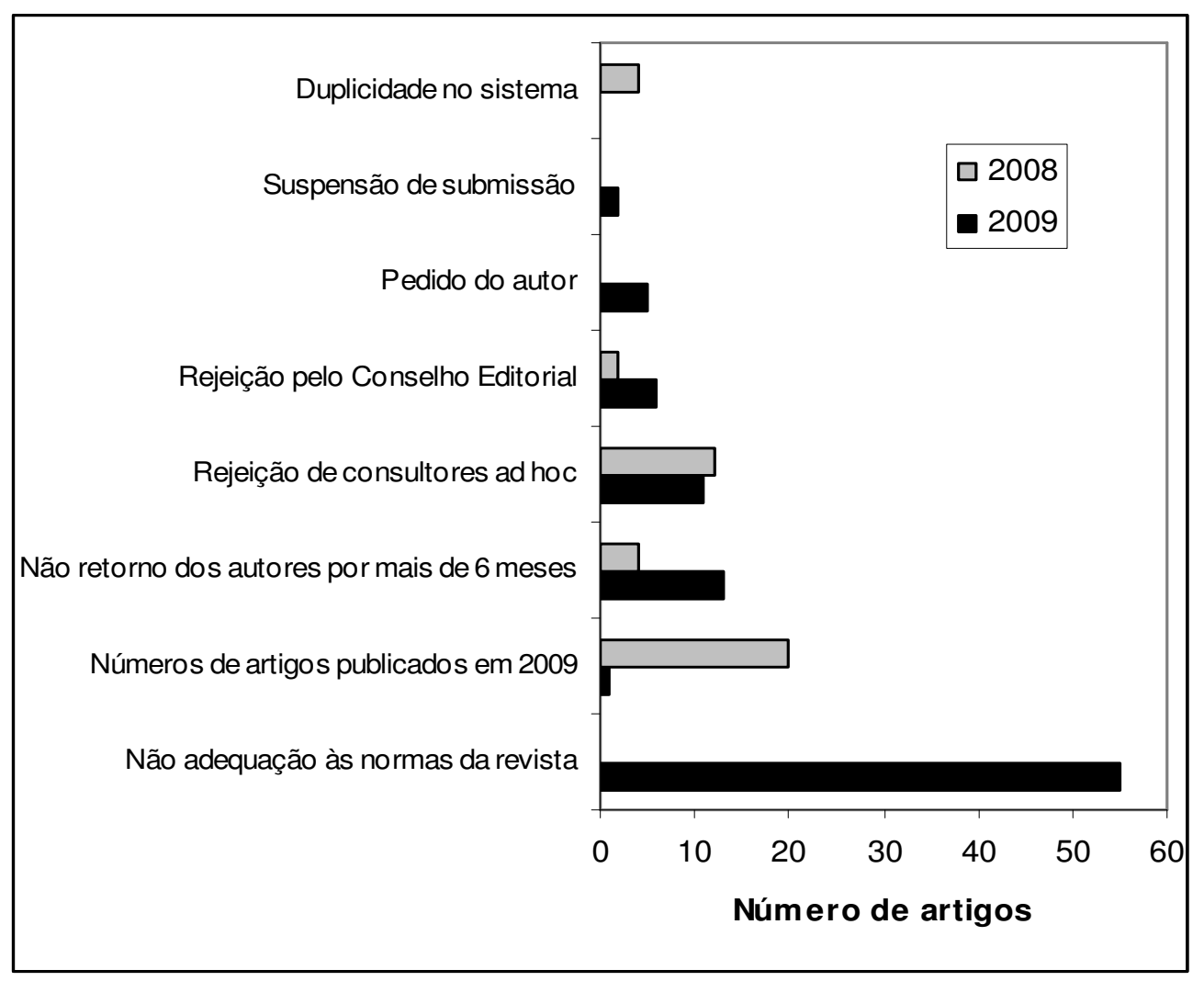

Figura 8. Número de Artigos Submetidos em 2008 ou 2009 em Relação aos Motivos de Arquivamento em 2009

A busca pela qualidade e pela resolução dos problemas surgidos tem possibilitado o aperfeiçoamento dos procedimentos de tramitação, arbitragem e normas de publicação, que se encontram regularmente em todos os números desde 2000, assumindo a forma de instruções aos autores. No ano de 2007, todos os itens, com exceção do fluxograma de publicação, tiveram alterações visando ao aprimoramento da publicação, e em 2009 as normas foram revisadas novamente, em decorrência das exigências postas pelo sistema Scielo e pelas submissões on-line, buscando torná-las ainda mais detalhadas, com vista a minimizar dúvidas dos autores e agilizar o processo de editoração.
A revista Psicologia em Estudo conta ainda com um sistema de distribuição do periódico que compreende permutas, doações, vendas e assinaturas, funcionando da seguinte forma: doação de um exemplar de cada número para as IESs públicas que oferecem o curso de Psicologia; permutas de periódicos classificados com conceitos $\mathrm{A}, \mathrm{B}$ e $\mathrm{C}$ em âmbito nacional, podendo ser de IESs públicas ou privadas; vendas, por assinatura ou de números avulsos, às IESs privadas que não têm como estabelecer permuta com o nosso periódico; doação de dois exemplares aos autores de cada artigo publicado; doação de um exemplar de cada número para indexadores, membros do Conselho Editorial e 
Conselhos de Psicologia; e cortesias esporádicas em eventos científicos da área de Psicologia.

No ano de 2009, por exemplo, foram realizadas permutas com 45 periódicos brasileiros, os quais fazem parte do acervo da biblioteca da Universidade Estadual de Maringá. A revista também é doada às bibliotecas de 58 universidades públicas do Brasil e do Exterior que têm o curso de graduação em Psicologia. Dessas instituições, 21 têm programa de pósgraduação em Psicologia, o que demonstra sua contribuição efetiva na formação de profissionais da área de Psicologia e afins, em nível de graduação e pós-graduação.

O Conselho Editorial do periódico é composto de 19 profissionais reconhecidos de universidades nacionais e internacionais, os quais participam das discussões sobre a dinâmica da editoração, quando solicitados. Além disso, a revista Psicologia em Estudo possui um banco de dados de consultores $a d$ hoc, com aproximadamente 340 nomes cadastrados, a maioria com máxima titulação acadêmica - doutores e pós-doutores - de Universidades de diferentes Estados da federação brasileira, bem como internacionais. Estes profissionais se dispõem a ler e reler (no caso de reformulações), avaliar e emitir um parecer técnico/teórico sobre os textos que recebemos, e desta forma prestam um importante serviço para garantir a qualidade de nossa publicação. No final deste relatório será apresentada a nominata dos consultores ad hoc que foram nossos colaboradores em 2009.

É importante destacar que, não obstante as dificuldades enfrentadas na editoração e publicação em uma universidade pública estadual - no caso, a Universidade Estadual de Maringá, no Interior do Paraná - que muitas vezes não dispõe de verbas suficientes para sua manutenção, ainda assim a revista Psicologia em Estudo vem conseguindo manter-se dentro dos requisitos exigidos em nível nacional e internacional. Além disso, a Revista privilegia publicações que enfatizem o aprofundamento teórico; assim muitos artigos são oriundos de teses $\mathrm{e}$ dissertações, tendo prioridade as pesquisas qualitativas na área de Ciências Humanas, o que garante também sua intersecção com outras áreas além da Psicologia.

\section{NOMINATA DE CONSULTORES 2009}

Acácia Aparecida Angeli dos Santos (Universidade São Francisco)

Adriano Furtado Holanda (UFPR)

Alessandra Ramos Castanha (Faculdade Boa Viagem PE)
Alice Maggi (Universidade de Caxias do Sul)

Alysson Massote Carvalho (Instituto Presbiteriano Gammon)

Ana Laura Schliemann (PUC-SP)

Ana Lúcia Queiroz Bezerra (Universidade Federal de Goiás)

Ana Maria de Oliveira Cintra (Universidade Federal de São João Del Rei)

Ana Maria Kirschner (UFF)

Anamélia Lins e Silva Franco (Universidade Católica de Salvador)

André Leclerc (UFPB)

Andréa Vieira Zanella (UFSC)

Angela Maria Cristina Uchoa de Abreu Branco (UNB)

Angélica Bastos de Feitas Rachid Grimberg (UFRJ)

Antonio Roberto Rocha Santos (UFPE)

Bianca Maria Sanches Faveret (UFJF)

Carla Ribeiro Guedes (UERJ)

Carlo Schmidt (Universidade Regional Integrada do Alto Uruguai e das Missões)

Carlos Eduardo Lopes (UEM)

Carlos Nazareno Ferreira Borges (UFES)

Carmen Lucia Guimarães de Mattos (UERJ)

Carmen Lucia Cardoso (USP)

Carmen Maria Bueno Neme (UNESP/Bauru)

Cássio Adriano Braz de Aquino (UFCE)

Cassio Rodrigues (Universidade Bandeirante de São Paulo)

Charles Elias Lang (UFAL)

Claire Terezinha Lazzaretti (UFPR)

Cleomar Azevedo (Centro Universitário FIEO)

Conceição Aparecida Serralha (Universidade Federal do Triângulo Mineiro)

Cristina Moreira Marcos (PUC-MG)

Daniel Abs (UFRS)

Daniela Cristina Sampaio de Brito (Psicóloga do Hospital Público Regional de Betim)

Daniele Nunes Henrique Silva (UNB)

Dayse Stoklos Malucelli (Universidade Tuiuti -PR)

Dirce Stein Backes (UFSC)

Edna Maria Querido de Oliveira Chamon (UNITAU)

Edna Martins (Universidade Ibirapuera)

Eduardo José Manzini (UNESP-Marília)

Elaine Soares Neves Lange (Universidade São Francisco)

Eliana Marcello De Felice (USF - UNIP - Mackenzie)

Eliane Robert Moraes (PUC-SP)

Elieuza Aparecida de Lima (UFU)

Elizabeth Ferreira Linhares (UFRJ)

Ellika Trindade (PUC/Minas)

Emerson Fernando Rasera (UFU) 
Ester Eliane Jeunon (Faculdade Novos Horizontes)

Eulália Maria Chaves Maia (UFRN)

Fabián Javier Marín Rueda (Universidade São Francisco)

Georges Daniel Janja Bloc Boris (UNIFOR)

Giana Bitencourt Frizzo (UFRGS)

Gleiber Couto Santos (Faculdade de Estudos Administrativos de Minas Gerais)

Gustavo Adolfo de Mello Neto (UEM)

Helga Loos (UFPR)

Heliane de Almeida Lins Leitão (UFAL)

Heloísa Benevides de Carvalho Chiattone (PUC-SP)

Hilma Tereza Tôrres Khoury (UFPA)

Ione Collado Pacheco Dourado (Faculdades Oswaldo Cruz)

Iracema Neno Cecilio Tada (UFRO)

Isabel Cristina Bonadio (USP)

Izabel Cristina Ferreira Borsoi (UFES)

Janaína Bianca Barletta (Universidade Tiradentes)

João Carlos Alchieri (UFRN)

João de Fernandes Teixeira (UFSC)

João Gabriel Lima Cruz Teixeira (UNB)

Jonas Fornitano Cholfe (Universidade Metodista de Piracicaba)

Jorge Luiz Cardoso Lyra-da-Fonseca (Instituto Papai PE)

José Antônio Damásio Abib (UEM)

Juliana Perucchi (UFJF)

Karina Pereira Pinto (Universidade Estadual de Santa Cruz)

Kely Cesar Martins de Paiva (Unihorizontes)

Leía Priszkulnik (USP)

Leila Maria Amaral Ribeiro (UFRJ)

Lia Carneiro Silveira (UECE)

Lígia Márcia Martins UNESP/Bauru)

Liza Fensterseifer (PUC-MG)

Lucia Cecília da Silva (UEM)

Lúcia de Mello e Souza Lehmann(UFF)

Lucia Emmanoel Novaes Malagris (UFRJ)

Lucia Helena de Feitas Pinho França (UNIVERSO)

Luciana Suárez Grzybowski (UNOCHAPECO)

Luís Fernando Tófoli (UFCE)

Luziane Zacche Avellar (UFES)

Maddi Damiao Junior (Secretaria de saúde de Vassouras)

Manoel Antônio dos Santos (USP)

Marcia Andrade (UNIFIEO)

Márcia Stengel (PUC-MG)

Marco Antonio Pereira Teixeira (UFRGS)

Marco Antônio Rotta Teixeira (UEM)

Marcus Tulio Caldas (Universidade Católica de Pernambuco)
Maria Abigail Souza (USP)

Maria Aparecida Crepaldi (UFSC)

Maria Cecília Maringoni de Carvalho (UFPI)

Maria Cristina Antunes (USP)

Maria Cristina Chimelo Paim (Universidade Luterana do Brasil)

Maria da Graça Correa Jacques (UFRGS)

Maria das Gracas Torres da Paz (Universidade Católica de Brasília)

Maria do Carmo Borges de Souza (UFRJ)

Maria Elenita Corrêa de Sampaio Favarato (USP)

Maria Jesia Vieira (UFSE)

Maria Júlia Lemes Ribeiro (UEM)

Maria Lucia Rocha-Coutinho (UFSC)

Maria Luisa Mendes Teixeira (Universidade Presbiteriana Mackenzie)

Maria Luiza Puglisi Munhoz (UNIFEO)

Maria Sylvia Cardoso Carneiro (UFSC)

Mariana Luisa Garcia Braido (PUC-RJ)

Marina de Bittencourt Bandeira (UDSJ)

Marines Saraiva (UEM)

Mario Sergio Ribeiro (UFJF)

Marlene Fagundes Carvalho Gonçalves (USP)

Marli Aparecida Calça Sanches (UEM)

Marta Regina de Leão D'Agord (UFRGS)

Mary Garcia Castro (Universidade Católica do Salvador)

Mary Sandra Carlotto (ULBRA-RS)

Monalisa Muniz Nascimento (Universidade São Francisco)

Nádia Eidt (UNESP-Bauru)

Nely Maria dos Santos Castro (Faculdade São Camilo - MG)

Nilton Soares Formiga (Universidade Federal da Paraiba)

Patrícia Alvarenga (UFBA)

Patrícia Kuerten Rocha (UFSC)

Patricia Valle de Albuquerque Lima (Instituto Carioca de Gestalt-terapia)

Paula Carolina Barboni Dantas Nascimento (USP RP)

Paula Inez Cunha Gomide (Universidade Tuiuti - PR)

Paulo Afranio Sant'Anna (Mackenzie)

Paulo César Ribeiro Martins (UPF/RS)

Rafaela Teixeira Zorzanelli (Fiocruz)

Raquel de Fari Brino (UFSCar)

Regina Perez Christofolli Abeche (UEM)

Regina Taam (UEM)

Renata Fabiana Pegoraro (UNESP)

Renata Mattos de Azevedo (UERJ)

Roberta Stubs Parpinelli (UEM)

Roberto Araujo Bello (UFRJ)

Roberto Patrus Mundim Pena (PUC-MG) 
Rodrigo Sanches Peres (UFU)

Rogério Rodrigues da Silva (Conselho da Justiça Federal $-\mathrm{DF})$

Rosa Guedes Lopes (Universidade Estácio de Sá)

Rozilda das Neves Alves (UEM)

Sheila Gonçalves Câmara (ULBRA/Canoas)

Sheila Regina de Camargo Martins (UEM)

Silvana Carneiro Maciel (UFPB)

Silvia Pereira da Cruz Benetti (UNISINOS)

Silvio Yasui (UNESP-Assis)

Simone da Nóbrega Tomaz Moreira (Centro de Saúde

Anita Garibaldi do Instituto Internacional de

Neurociências)

Simone Moreira de Moura (UEL)

Simone Ouvinha Peres (UFRJ)
Sonia Regina Loureiro (USP)

Sueli Aparecida Freire (UFU)

Sueli Ferreira (Universidade de Uberaba)

Suzane Schmidlin Löhr (UFPR)

Sylvia Mara Pires de Freitas (UEM)

Tatiana Ramminger (Fundação Oswaldo Cruz)

Tereza Cristina Cavalcanti Ferreira de Araujo (UNB)

Ued Martins Manjud Maluf (UFRJ)

Vânia Lúcia Sant'Ana Pestana (UEM)

Vania Nora Bustamante Dejo (London School of Economics)

Viviana Carola Velasco Martinez (UEM) 
